\title{
THE GENERAL EXACT SOLUTION FOR THE MANY MOMENTS MACROSCOPIC APPROACH TO EXTENDED THERMODYNAMICS OF POLYATOMIC GASES
}

\author{
M.C. Carrisi ${ }^{1}$, R. Enoh Tchame ${ }^{2}$, \\ M. Obounou ${ }^{3}$, S. Pennisi ${ }^{4} \S$ \\ ${ }^{1,4}$ Department of Mathematics and Informatics \\ University of Cagliari \\ Via Ospedale 72, 09124 Cagliari, ITALY \\ ${ }^{2,3}$ Department of Physics \\ University of Yaoundé I \\ Yaoundé, CAMEROON
}

\begin{abstract}
A new model for Polyatomic and for Dense Gases has been proposed in literature in the last five years in the framework of Extended Thermodynamics. The case with an arbitrary but fixed number of moments has been recently studied, both with the kinetic approach than with the macroscopic approach; this last one is more general and includes the results of the kinetic approach only as a particular case.

Scope of the "closure problem" is to find the expression of some arbitrary functions which appear in the balance equations. Up to now only a recurrence procedure has been published which outlines how to find the solution of this problem with the macroscopic approach; by using this procedure, a numberable set of solutions has been found and written explicitly, while we find here the most general exact solution. It is determined except for some arbirary terms and it is interesting that these terms appear also in the 24 moments model; so we find here that they are transmitted from the model with 24 moments to those with an arbitrary number of moments, without any further arbitrary term.
\end{abstract}

AMS Subject Classification: $74 \mathrm{~A} 15,76 \mathrm{~N} 15,80 \mathrm{~A} 17$

Key Words: thermodynamics, gas dynamics, thermodynamics of continua

Received: October 15, 2016

Revised: $\quad$ February 15, 2017

Published: February 19, 2017

${ }^{\S}$ Correspondence author (c) 2017 Academic Publications, Ltd. url: www.acadpubl.eu 


\section{Introduction}

Extended Thermodynamics of Polyatomic Gases (ETDG) with many moments proposes for every number $N$ the following balance equations

$$
\begin{aligned}
& \partial_{t} F^{i_{1} \cdots i_{n}}+\partial_{k} F^{k i_{1} \cdots i_{n}}=P^{i_{1} \cdots i_{n}} \quad \text { for } n=0, \cdots N+2 . \\
& \partial_{t} G^{i_{1} \cdots i_{m}}+\partial_{k} G^{k i_{1} \cdots i_{m}}=Q^{i_{1} \cdots i_{m}}
\end{aligned}
$$

and the resulting model is called an $N$ - Model (See [1]-[4] ).

Another possibility is that the index $m$ in eq. $(1)_{2}$ goes from 0 to an arbitrary number $M$ restricted by $M \leq N+1$ and the resulting model can be called the $(N-2, M)$ - Model as in [5] which belongs to the kinetic approach. But this apparently more general model can be obtained from the present one in the following way:

- Let us consider firstly the eqs. (1) with $N+M$ instead of $N$, that is the $(N+M)-$ Model .

- Let us consider now the subsystem obtained from the above one by simply removing from $(1)_{1}$ the equations for $n=N+3, \cdots N+M+2$ and from $(1)_{2}$ the equations for $m=M+1, \cdots N+M$; this can be done by simply putting equal to zero the corresponding Lagrange multipliers according to the technique outlined in [6].

In this way, the $(N-2, M)$ - Model is recovered starting from the present one. Now, always in [5], it is shown that in the context of the kinetic approach is necessary to choose $N=M-1$ if we want characterisic velocities depending on the degrees of freedom of a molecule. This is not a problem because we can proceed in the following way:

Let us consider the subsystem obtained by simply removing from $(1)_{1}$ the equation for $n=N+2$; this can be done by simply putting equal to zero the corresponding Lagrange multiplier according to the technique outlined in [6]. In this way the number of the eqs. $(1)_{2}$ will be equal to that of $(1)_{1}$ decreased of an unity obtaining in this way the same set of equations of [5].

In conclusion, we may consider the $N$ - Model without loss of generality and this choice is motivated by the fact that within the macroscopic approach this model is easier to handle.

Before going on, we now recall that the present model belongs to the framework of Extended Thermodynamics (ET) which started with the articles [7], [8] and produced many other articles the majority of which are reported in the 
book [9]. It used only eqs. (1) $)_{1}$ obtaining the important result to have a symmetric hyperbolic system of partial differential equations, with finite speed of propagation of shock waves and other important properties. But the structure of ET implied the restriction $p=\frac{2}{3} \rho \epsilon$, where $p$ is the equilibrium pressure and $\epsilon$ the internal energy. This problem have been overcome in ETDG by considering all the balance equations (1) of which the first one is called the "Mass-Block" of equations and the second is called the "Energy-Block" of equations.

The first article on this subject is [10] and, after that, a prolific production followed; we can read in the references of the book [11] the majority of the subsequent articles on this subject. The last significative aricle on this subject is [12], while the contribute of some of the present author in this framework are [13]-[17] besides those cited above. In (1) the independent variables are $F^{i_{1} \cdots i_{n}}$ and $G^{i_{1} \cdots i_{m}}$; the quantities $F^{k i_{1} \cdots i_{n}}$ and $G^{k i_{1} \cdots i_{m}}$ are their corresponding fluxes. We see that each flux is equal to the independent variable of the subsequent equation, except for the flux in the last equation of the Mass-Block and for that in the last equation of the Energy-Block; for these last fluxes we know only that they are symmetric tensors. We will refer to this property as "the symmetry conditions". The problem will be closed when we know the expressions of $F^{k i_{1} \cdots i_{N+2}}$ and $G^{k i_{1} \cdots i_{N}}$ as functions of the independent variables. Restrictions on their generality are obtained by imposing the entropy principle, the Galilean relativity principle and the symmetry conditions.

The Entropy Principle can be exploited through Liu's Theorem [18] and by using a bright idea conceived by Ruggeri [19]; so it becomes equivalent to assuming the existence of Lagrange Multipliers $\mu_{A}$ and $\lambda_{B}$ which can be taken as independent variables and, after that, we have

$$
\begin{aligned}
& F^{i_{1} \cdots i_{n}}=\frac{\partial h^{\prime}}{\partial \mu_{i_{1} \cdots i_{n}}} \quad, \quad G^{i_{1} \cdots i_{m}}=\frac{\partial h^{\prime}}{\partial \lambda_{i_{1} \cdots i_{m}}} \\
& F^{k i_{1} \cdots i_{n}}=\frac{\partial h^{\prime k}}{\partial \mu_{i_{1} \cdots i_{n}}} \quad, \quad G^{k i_{1} \cdots i_{m}}=\frac{\partial h^{\prime k}}{\partial \lambda_{i_{1} \cdots i_{m}}} .
\end{aligned}
$$

which expresses all the moments in terms of only two unknown functions, the 4-potentials $h^{\prime}, h^{\prime k}$. A nice consequence of eqs. (2) is that the field equations assume the symmetric form. Another consequence of (2) is that the above mentioned symmetry conditions and the Galilean Relativity Principle can be expressed as

$$
\frac{\partial h^{\prime k}}{\partial \mu_{i_{1} \cdots i_{n}}}=\frac{\partial h^{\prime}}{\partial \mu_{k i_{1} \cdots i_{n}}} \quad \text { for } n=0, \cdots, N+1 ; \frac{\partial h^{\prime[k}}{\partial \mu_{\left.i_{1}\right] i_{2} \cdots i_{N+2}}}=0
$$




$$
\begin{aligned}
& \frac{\partial h^{\prime k}}{\partial \lambda_{i_{1} \cdots i_{m}}}=\frac{\partial h^{\prime}}{\partial \lambda_{k i_{1} \cdots i_{m}}} \text { for } m=0, \cdots, N-1 ; \quad \frac{\partial h^{\prime[k}}{\partial \lambda_{\left.i_{1}\right] \cdots i_{N}}}=0 . \\
& \frac{\partial h^{\prime}}{\partial \mu_{k}} \mu_{i}+\sum_{n=1}^{N+1} \frac{\partial h^{\prime}}{\partial \mu_{k j_{1} \cdots j_{n}}}\left[(n+1) \mu_{i j_{1} \cdots j_{n}}+2 \lambda_{j_{1} \cdots j_{n-1}} \delta_{j_{n}}\right]+ \\
& +\sum_{s=0}^{N-1} \frac{\partial h^{\prime}}{\partial \lambda_{k h_{1} \cdots h_{s}}}(s+1) \lambda_{i h_{1} \cdots h_{s}}+h^{\prime} \delta^{k i}=0 .
\end{aligned}
$$

We don' t go in the details on how the the Galilean Relativity Principle, thanks also to the other conditions, is equivalent to $(3)_{5}$; this equivalence can be already found in literature. In the next section, eqs. (4) and (5), we will exhibit a particular but significative solution of the conditions (3), which is expressed through a Taylor' s expansion around equilibrium; this is defined as the state where $\mu_{i_{1} \cdots i_{n}}=0$ for $n=1, \cdots, N+2, \lambda_{i_{1} \cdots i_{m}}=0$ for $m=1, \cdots, N$, so that the only variables which are not zero at equilibrium are $\mu$ and $\lambda$. The first of these is the chemical potential, while $\lambda=\frac{1}{2 T}$ with $T$ absolute temperature.

In sect. 3 we will report a part of the proof that eqs. (4) and (5) give a solution of (3). The second and final part of the proof is described in sect. 4 .

\section{An Exact Solution of the Conditions (3).}

Before writing our solution we need to describe an hard but straightforward notation:

To do the derivatives with respect to $\mu_{i_{1} \cdots i_{n}}$ a number $p_{n}$ of times, we use the compact form $A_{n, 1}$ to indicate a set of $n$ indexes, $A_{n, 2}$ to indicate another set of $n$ indexes, and so on up to $A_{n, p_{n}}$. For example,

$$
\begin{aligned}
& \frac{\partial^{p_{2}} h^{\prime k}}{\partial \mu_{h_{1} k_{1}} \partial \mu_{h_{2} k_{2}} \cdots \partial \mu_{h_{p_{2}} k_{p_{2}}}} \text { in the compact notation becomes } \\
& \frac{\partial^{p_{2}} h^{\prime k}}{\partial \mu_{A_{2,1}} \partial \mu_{A_{2,2}} \cdots \partial \mu_{A_{2, p_{2}}}}
\end{aligned}
$$

because the first index of $A \ldots, \ldots$ indicates how many indexes has the corresponding $\mu \ldots$, while the second index of $A \ldots, \ldots$ indicates how many derivatives we are taking with respect to it.

$$
\text { Similarly, } \frac{\partial^{p_{3}} h^{\prime k}}{\partial \mu_{h_{1} k_{1} j_{1}} \partial \mu_{h_{2} k_{2} j_{2}} \cdots \partial \mu_{h_{p_{3}} k_{p_{3}} j_{p_{3}}}} \text { becomes }
$$




$$
\frac{\partial^{p_{3}} h^{\prime k}}{\partial \mu_{A_{3,1}} \partial \mu_{A_{3,2}} \cdots \partial \mu_{A_{3, p_{3}}}}
$$

and so on. By using this notation, we can now write our solution and it is

$$
\begin{aligned}
& h^{\prime}=\sum_{p_{0}, \cdots, p_{N+2}, r_{1}, \cdots, r_{N}}^{0 \cdots \infty} \frac{1}{p_{0} !} \frac{1}{p_{1} !} \cdots \frac{1}{p_{N+2} !} \frac{1}{r_{1} !} \frac{1}{r_{2} !} \cdots \frac{1}{r_{N} !} . \\
& \sum_{i=1}^{N+2} i p_{i}+\sum_{i=1}^{N} i r_{i} \text { even } \\
& \cdot \frac{\left[1+\sum_{i=1}^{N+2} i p_{i}+\sum_{i=1}^{N} i r_{i}\right] ! !}{1+\sum_{i=1}^{N+2} i p_{i}+\sum_{i=1}^{N} i r_{i}} \frac{1+\sum_{i=0}^{N+2}(i-2) p_{i}+\sum_{i=1}^{N} i r_{i}}{\left[1+\sum_{i=0}^{N+2}(i-2) p_{i}+\sum_{i=1}^{N} i r_{i}\right] ! !} . \\
& \cdot\left\{\sum_{r_{0}=1}^{\infty} C_{\left(\sum_{i=0}^{N+2} p_{i}-r_{0},\right.} \sum_{i=0}^{N+2}(i-2) p_{i}+\sum_{i=1}^{N} i r_{i}-1\right) \frac{\partial^{\sum_{i=1}^{N} r_{i}}}{\partial \lambda^{\sum_{i=1}^{N} r_{i}}}\left[\left(\frac{-1}{2 \lambda}\right)^{r_{0}}\right]+ \\
& +\sum_{r_{0}=0}^{\infty} \frac{1}{r_{0} !}(-2)^{\sum_{i=0}^{N} r_{i}} \\
& \left.\cdot\left(\sum_{i=0}^{N} r_{i}\right) ! C_{\left(\sum_{i=0}^{N+2} p_{i}+\sum_{i=0}^{N} r_{i} \quad, \quad \sum_{i=0}^{N+2}(i-2) p_{i}+\sum_{i=1}^{N} i r_{i}-1\right)}(\lambda)^{r_{0}}\right\} \text {. } \\
& \cdot \delta^{\left(A_{1,1} \cdots A_{1, p_{1}} \cdots A_{N+2,1} \cdots A_{N+2, p_{N+2}} B_{1,1} \cdots B_{1, r_{1}} \cdots B_{N, 1} \cdots B_{N, r_{N}}\right)}(\mu)^{p_{0}} . \\
& \mu_{A_{1,1}} \cdots \mu_{A_{1, p_{1}}} \cdots \mu_{A_{N+2,1}} \cdots \mu_{A_{N+2, p_{N+2}}} . \\
& \cdot \lambda_{B_{1,1}} \cdots \lambda_{B_{1, r_{1}}} \cdots \lambda_{B_{N, 1}} \cdots \lambda_{B_{N, r_{N}}} \\
& h^{\prime k}=\sum_{p_{0}, \cdots, p_{N+2}, r_{1}, \cdots, r_{N}}^{0 \cdots \infty} \frac{1}{p_{0} !} \frac{1}{p_{1} !} \cdots \frac{1}{p_{N+2} !} \frac{1}{r_{1} !} \frac{1}{r_{2} !} \cdots \frac{1}{r_{N} !} . \\
& \sum_{i=1}^{N+2} i p_{i}+\sum_{i=1}^{N} i r_{i} \text { odd }
\end{aligned}
$$




$$
\begin{aligned}
& \cdot \frac{\left[\sum_{i=1}^{N+2} i p_{i}+\sum_{i=1}^{N} i r_{i}\right] ! !}{\left[\sum_{i=0}^{N+2}(i-2) p_{i}+\sum_{i=1}^{N} i r_{i}\right] ! !} \\
& \cdot\left\{\sum_{r_{0}=1}^{\infty} C_{\left(\sum_{i=0}^{N+2} p_{i}-r_{0}\right.} \quad, \quad \sum_{i=0}^{N+2}(i-2) p_{i}+\sum_{i=1}^{N} i r_{i}\right) \frac{\partial^{\sum_{i=1}^{N} r_{i}}}{\partial \lambda^{\sum_{i=1}^{N} r_{i}}}\left[\left(\frac{-1}{2 \lambda}\right)^{r_{0}}\right]+ \\
& +\sum_{r_{0}=0}^{\infty} \frac{1}{r_{0} !}(-2)^{\sum_{i=0}^{N} r_{i}}\left(\sum_{i=0}^{N} r_{i}\right) ! \cdot \\
& \left.\cdot C_{\left(\sum_{i=0}^{N+2} p_{i}+\sum_{i=0}^{N} r_{i} \quad, \quad \sum_{i=0}^{N+2}(i-2) p_{i}+\sum_{i=1}^{N} i r_{i}\right)}(\lambda)^{r_{0}}\right\} . \\
& \cdot \delta^{\left(k A_{1,1} \cdots A_{1, p_{1}} \cdots A_{N+2,1} \cdots A_{N+2, p_{N+2}} B_{1,1} \cdots B_{1, r_{1}} \cdots B_{N, 1} \cdots B_{N, r_{N}}\right)}(\mu)^{p_{0}} . \\
& \cdot \mu_{A_{1,1}} \cdots \mu_{A_{1, p_{1}}} \cdots \mu_{A_{N+2,1}} \cdots \mu_{A_{N+2, p_{N+2}}} \cdot \\
& \cdot \lambda_{B_{1,1}} \cdots \lambda_{B_{1, r_{1}}} \cdots \lambda_{B_{N, 1}} \cdots \lambda_{B_{N, r_{N}}}
\end{aligned}
$$

where $C_{r, s}$ is a family of constants such that $C_{r, s}=0$ if at least one of the indexes $r, s$ is negative or if $r>s$. Despite the appearance, these expressions are not complicated. In fact, the factorial $\frac{1}{p_{1} !}$ appears also in the Taylor' s expansions of functions depending on a single variable; we have here one of these for every variable, both of the mass block than of the energy block. Moreover, in these expressions appears the sum of these numbers $p_{1}+p_{2}+\cdots+p_{N+2}$ of the mass block and $r_{1}+r_{2}+\cdots+r_{N}$ of the energy block. Moreover, these numbers appear also through $p_{1}+2 p_{2}+\cdots+(N+2) p_{N+2}+r_{1}+2 r_{2}+\cdots+N r_{N}$ where these numbers are multiplied by the order of the Lagrange multiplier which they represent; after that, their sum is taken. The condition " $p_{1}+2 p_{2}+\cdots+$ $(N+2) p_{N+2}+r_{1}+2 r_{2}+\cdots+N r_{N}$ odd" in the expression of $h^{\prime k}$ is necessary because the following $\delta^{\cdots}$ must have an even number of indexes; similarly, for the expression of $h^{\prime}$. Here we find only a letter $\delta^{\cdots}$ but it is understood that it is a shortened symbol denoting the product of some $\delta^{\cdots}$ each one with 2 indexes, and with a final symmetrization over all these indexes. Finally, in the last line there is the product of the variables with respect to which we have done the Taylor' s expansions. 


\section{Proof of the Solution (4) and (5) - Part I}

We prove now that $(4),(5)$ is a solution of $(3)_{1-4}$, while $(3)_{5}$ will be considered in the next section.

- Let us begin with $(3)_{1}$.

To verify it we have to take into account that $\mu_{i_{1} \cdots i_{n}}$ here is denoted with $\mu_{A_{n, 1}}$, or with $\mu_{A_{n, 2}}$, and so on up to $\mu_{A_{n, p_{n}}}$. Similarly, $\mu_{k i_{1} \cdots i_{n}}$ is denoted with $\mu_{A_{n+1,1}}$, or with $\mu_{A_{n+1,2}}$, and so on up to $\mu_{A_{n+1, p_{n+1}}}$. Consequently, the left hand side of $(3)_{1}$ causes, with respect to the expression (5), a rise of one unity of the index $p_{n}$; similarly, the right hand side of $(3)_{1}$ causes, with respect to the expression (4), a rise of one unity of the index $p_{n+1}$. More precisely, we obtain for both sides the following expression

$$
\begin{aligned}
& \sum^{0 \cdots \infty} \\
& p_{0}, \cdots, p_{N+2}, r_{1}, \cdots, r_{N} \\
& \frac{1}{p_{0} !} \frac{1}{p_{1} !} \cdots \frac{1}{p_{N+2} !} \frac{1}{r_{1} !} \frac{1}{r_{2} !} \cdots \frac{1}{r_{N} !} . \\
& n+\sum_{i=1}^{N+2} i p_{i}+\sum_{i=1}^{N} i r_{i} \text { odd } \\
& \cdot \frac{\left[n+\sum_{i=1}^{N+2} i p_{i}+\sum_{i=1}^{N} i r_{i}\right] ! !}{\left[n-2+\sum_{i=0}^{N+2}(i-2) p_{i}+\sum_{i=1}^{N} i r_{i}\right] ! !}
\end{aligned}
$$

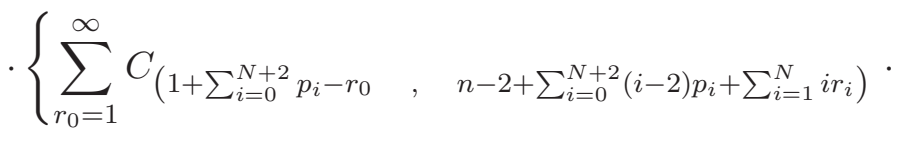

$$
\begin{aligned}
& \cdot \frac{\partial^{\sum_{i=1}^{N} r_{i}}}{\partial \lambda^{\sum_{i=1}^{N} r_{i}}}\left[\left(\frac{-1}{2 \lambda}\right)^{r_{0}}\right]+\sum_{r_{0}=0}^{\infty} \frac{1}{r_{0} !}(-2)^{\sum_{i=0}^{N} r_{i}}\left(\sum_{i=0}^{N} r_{i}\right) ! \cdot \\
& \text { - } \left.C_{\left(1+\sum_{i=0}^{N+2} p_{i}+\sum_{i=0}^{N} r_{i} \quad, \quad n-2+\sum_{i=0}^{N+2}(i-2) p_{i}+\sum_{i=1}^{N} i r_{i}\right)}(\lambda)^{r_{0}}\right\} \text {. } \\
& \cdot \delta^{\left(k i_{1} \cdots i_{n} A_{1,1} \cdots A_{1, p_{1}} \cdots A_{N+2,1} \cdots A_{N+2, p_{N+2}} B_{1,1} \cdots B_{1, r_{1}} \cdots B_{N, 1} \cdots B_{N, r_{N}}\right)}(\mu)^{p_{0}} \cdot \\
& \cdot \mu_{A_{1,1}} \cdots \mu_{A_{1, p_{1}}} \cdots \mu_{A_{N+2,1}} \cdots \mu_{A_{N+2, p_{N+2}}} \cdot \\
& \cdot \lambda_{B_{1,1}} \cdots \lambda_{B_{1, r_{1}}} \cdots \lambda_{B_{N, 1}} \cdots \lambda_{B_{N, r_{N}}} \cdot
\end{aligned}
$$


- It is easy to verify $(3)_{2,4}$.

- Let us continue verifying $(3)_{3}$ in the case $m \geq 1$.

To verify it we have to take into account that $\lambda_{i_{1} \cdots i_{m}}$ here is denoted with $\lambda_{B_{m, 1}}$, or with $\lambda_{B_{m, 2}}$, and so on up to $\lambda_{B_{m, r_{m}}}$. Similarly, $\lambda_{k i_{1} \cdots i_{m}}$ is denoted with $\lambda_{B_{m+1,1}}$, or with $\lambda_{B_{m+1,2}}$, and so on up to $\lambda_{B_{m+1, r_{m+1}}}$. Consequently, the left hand side of $(3)_{3}$ with $m \geq 1$ causes, with respect to the expression (5), a rise of one unity of the index $r_{m}$; similarly, the right hand side of $(3)_{3}$ with $m \geq 1$ causes, with respect to the expression (4), a rise of one unity of the index $r_{m+1}$. More precisely, we obtain for both sides the following expression

$$
\begin{aligned}
& \sum^{0 \cdots \infty} \quad \frac{1}{p_{0} !} \frac{1}{p_{1} !} \cdots \frac{1}{p_{N+2} !} \frac{1}{r_{1} !} \frac{1}{r_{2} !} \cdots \frac{1}{r_{N} !} . \\
& p_{0}, \cdots, p_{N+2}, r_{1}, \cdots, r_{N} \\
& m+\sum_{i=1}^{N+2} i p_{i}+\sum_{i=1}^{N} i r_{i} \text { odd } \\
& \cdot \frac{\left[m+\sum_{i=1}^{N+2} i p_{i}+\sum_{i=1}^{N} i r_{i}\right] ! !}{\left[m+\sum_{i=0}^{N+2}(i-2) p_{i}+\sum_{i=1}^{N} i r_{i}\right] ! !}
\end{aligned}
$$

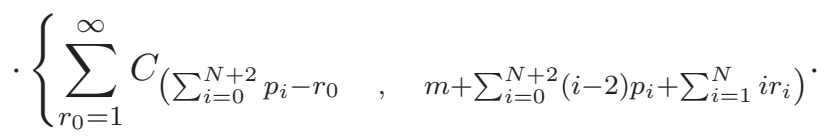

$$
\begin{aligned}
& \cdot \frac{\partial^{1+\sum_{i=1}^{N} r_{i}}}{\partial \lambda^{1+\sum_{i=1}^{N} r_{i}}}\left[\left(\frac{-1}{2 \lambda}\right)^{r_{0}}\right]+\sum_{r_{0}=0}^{\infty} \frac{1}{r_{0} !}(-2)^{1+\sum_{i=0}^{N} r_{i}}\left(1+\sum_{i=0}^{N} r_{i}\right) ! \cdot \\
& \left.\cdot C_{\left(1+\sum_{i=0}^{N+2} p_{i}+\sum_{i=0}^{N} r_{i}, m+\sum_{i=0}^{N+2}(i-2) p_{i}+\sum_{i=1}^{N} i r_{i}\right)}(\lambda)^{r_{0}}\right\} \cdot \\
& \cdot \delta^{\left(k i_{1} \cdots i_{m} A_{1,1} \cdots A_{1, p_{1}} \cdots A_{N+2,1} \cdots A_{N+2, p_{N+2}} B_{1,1} \cdots B_{1, r_{1}} \cdots B_{N, 1} \cdots B_{N, r_{N}}\right)}(\mu)^{p_{0}} \cdot \\
& \cdot \mu_{A_{1,1}} \cdots \mu_{A_{1, p_{1}}} \cdots \mu_{A_{N+2,1}} \cdots \mu_{A_{N+2, p_{N+2}}} \cdot \\
& \lambda_{B_{1,1}} \cdots \lambda_{B_{1, r_{1}}} \cdots \lambda_{B_{N, 1}} \cdots \lambda_{B_{N, r_{N}}}
\end{aligned}
$$

- Let us conclude verifying $(3)_{3}$ in the case $m=0$. 
We have the same situation of the above case for its right hand side, while for its left hand side we have to take simply the derivative of (5) with respect to $\lambda$. More precisely, we obtain for both sides the following expression

$$
\begin{aligned}
& \sum^{0 \cdots \infty} \\
& \frac{1}{p_{0} !} \frac{1}{p_{1} !} \cdots \frac{1}{p_{N+2} !} \frac{1}{r_{1} !} \frac{1}{r_{2} !} \cdots \frac{1}{r_{N} !} . \\
& p_{0}, \cdots, p_{N+2}, r_{1}, \cdots, r_{N} \\
& \sum_{i=1}^{N+2} i p_{i}+\sum_{i=1}^{N} i r_{i} \text { odd } \\
& \cdot \frac{\left[\sum_{i=1}^{N+2} i p_{i}+\sum_{i=1}^{N} i r_{i}\right] ! !}{\left[\sum_{i=0}^{N+2}(i-2) p_{i}+\sum_{i=1}^{N} i r_{i}\right] ! !} \cdot
\end{aligned}
$$

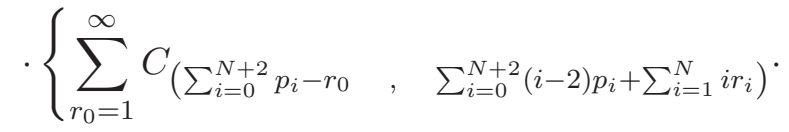

$$
\begin{aligned}
& \frac{\partial^{1+\sum_{i=1}^{N} r_{i}}}{\partial \lambda^{1+\sum_{i=1}^{N} r_{i}}}\left[\left(\frac{-1}{2 \lambda}\right)^{r_{0}}\right]+\sum_{r_{0}=0}^{\infty} \frac{1}{r_{0} !}(-2)^{1+\sum_{i=0}^{N} r_{i}}\left(1+\sum_{i=0}^{N} r_{i}\right) ! \cdot \\
& \text { - } \left.C_{\left(1+\sum_{i=0}^{N+2} p_{i}+\sum_{i=0}^{N} r_{i}, \sum_{i=0}^{N+2}(i-2) p_{i}+\sum_{i=1}^{N} i r_{i}\right)}(\lambda)^{r_{0}}\right\} \text {. } \\
& \cdot \delta^{\left(k A_{1,1} \cdots A_{1, p_{1}} \cdots A_{N+2,1} \cdots A_{N+2, p_{N+2}} B_{1,1} \cdots B_{1, r_{1}} \cdots B_{N, 1} \cdots B_{N, r_{N}}\right)}(\mu)^{p_{0}} . \\
& \cdot \mu_{A_{1,1}} \cdots \mu_{A_{1, p_{1}}} \cdots \mu_{A_{N+2,1}} \cdots \mu_{A_{N+2, p_{N+2}}} . \\
& \cdot \lambda_{B_{1,1}} \cdots \lambda_{B_{1, r_{1}}} \cdots \lambda_{B_{N, 1}} \cdots \lambda_{B_{N, r_{N}}}
\end{aligned}
$$

So we have finished to verify that $(4)$, (5) is a solution of $(3)_{1-4}$ and there remains to prove that it satisfies also $(3)_{5}$.

\section{Proof of the Solution (4) and (5) - Part II}

We prove now that $(4),(5)$ is a solution of $(3)_{5}$. To this regard we note firstly that in this condition we can put under an unique summation the coefficients of $\mu \ldots$, from the first coefficient of a $\lambda \ldots$ we can isolate the term with $n=1$ and in the other ones we can change the index of the summation according to the 
law $n=2+s$. In this way $(3)_{5}$ can be rewritten as

$$
\begin{gathered}
\sum_{n=0}^{N+1} \frac{\partial h^{\prime}}{\partial \mu_{k j_{1} \cdots j_{n}}}(n+1) \mu_{i j_{1} \cdots j_{n}}+\frac{\partial h^{\prime}}{\partial \mu_{k i}} 2 \lambda+\sum_{s=0}^{N-1} 2 \lambda_{j_{1} \cdots j_{s}+1} \frac{\partial h^{\prime}}{\partial \mu_{k j_{1} \cdots j_{s+1} i}}+ \\
+\sum_{s=0}^{N-1} \frac{\partial h^{\prime}}{\partial \lambda_{k h_{1} \cdots h_{s}}}(s+1) \lambda_{i h_{1} \cdots h_{s}}+h^{\prime} \delta^{k i}=0
\end{gathered}
$$

- Now, for the first term of this relation we can use (4) and the derivation causes a presence of the factor $p_{n+1}$, the substitution of $\mu_{A_{n+1,1}} \cdots \mu_{A_{n+1, p_{n+1}}}$ with $\mu_{A_{n+1,1}} \cdots \mu_{A_{n+1, p_{n+1}-1}}$ and the new free indexes $k j_{1} \cdots j_{n}$ in the expression of $\delta^{\cdots}$; more precisely, that term becomes

$$
\begin{aligned}
& \sum_{n=0}^{N+1}(n+1) \mu_{i j_{1} \cdots j_{n}} \frac{\partial h^{\prime}}{\partial \mu_{k j_{1} \cdots j_{n}}}=\sum_{n=0}^{N+1}(n+1) p_{n+1} \cdot \\
& \sum^{0 \cdots \infty} \quad \frac{1}{p_{0} !} \frac{1}{p_{1} !} \cdots \frac{1}{p_{N+2} !} \frac{1}{r_{1} !} \frac{1}{r_{2} !} \cdots \frac{1}{r_{N} !} . \\
& \begin{array}{c}
p_{0}, \cdots, p_{N+2}, r_{1}, \cdots, r_{N} \\
\sum_{i=1}^{N+2} i p_{i}+\sum_{i=1}^{N} i r_{i} \text { even }
\end{array} \\
& \cdot \frac{\left[1+\sum_{i=1}^{N+2} i p_{i}+\sum_{i=1}^{N} i r_{i}\right] ! !}{1+\sum_{i=1}^{N+2} i p_{i}+\sum_{i=1}^{N} i r_{i}} \frac{1+\sum_{i=0}^{N+2}(i-2) p_{i}+\sum_{i=1}^{N} i r_{i}}{\left[1+\sum_{i=0}^{N+2}(i-2) p_{i}+\sum_{i=1}^{N} i r_{i}\right] ! !}
\end{aligned}
$$

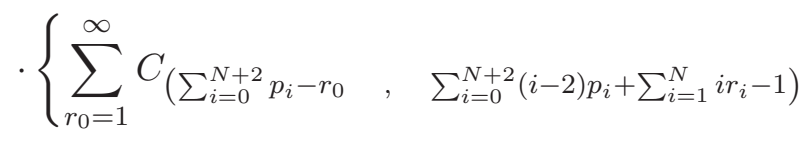

$$
\begin{aligned}
& \frac{\partial^{\sum_{i=1}^{N} r_{i}}}{\partial \lambda^{\sum_{i=1}^{N} r_{i}}}\left[\left(\frac{-1}{2 \lambda}\right)^{r_{0}}\right]+\sum_{r_{0}=0}^{\infty} \frac{1}{r_{0} !}(-2)^{\sum_{i=0}^{N} r_{i}}\left(\sum_{i=0}^{N} r_{i}\right) ! \cdot \\
& \left.\cdot C_{\left(\sum_{i=0}^{N+2} p_{i}+\sum_{i=0}^{N} r_{i} \quad, \quad \sum_{i=0}^{N+2}(i-2) p_{i}+\sum_{i=1}^{N} i r_{i}-1\right)}(\lambda)^{r_{0}}\right\} . \\
& \cdot \delta^{j_{n+1} i} \delta^{\left(k j_{1} \cdots j_{n} A_{1,1} \cdots A_{1, p_{1}} \cdots A_{n+1,1} \cdots A_{n+1, p_{n+1}-1} \cdots A_{N+2,1} \cdots A_{N+2, p_{N+2}}\right.}
\end{aligned}
$$




$$
\begin{array}{r}
\left.B_{1,1} \cdots B_{1, r_{1}} \cdots B_{N, 1} \cdots B_{N, r_{N}}\right) \\
\cdot(\mu)^{p_{0}} \mu_{A_{1,1}} \cdots \mu_{A_{1, p_{1}}} \cdots \mu_{A_{n+1,1}} \cdots \mu_{A_{n+1, p_{n+1}-1}} \\
\cdot \mu_{j_{1} \cdots j_{n+1}} \cdots \mu_{A_{N+2,1}} \cdots \mu_{A_{N+2, p_{N+2}}} \lambda_{B_{1,1}} \cdots \lambda_{B_{1, r_{1}}} \cdots \lambda_{B_{N, 1}} \cdots \lambda_{B_{N, r_{N}}}
\end{array}
$$

where we have also substituted $\mu_{i j_{1} \cdots j_{n}}$ with $\mu_{j_{1} \cdots j_{n+1}} \delta^{j_{n+1} i}$. Now in this expression we can insert a symmetrization over all the indexes of the set

$$
j_{1} \cdots j_{n+1} A_{n+1,1} \cdots A_{n+1, p_{n+1}-1}
$$

because that expression remains the same if we exchange two of these indexes. This fact is evident if the two indexes are taken between $j_{1} \cdots j_{n+1}$; for the proof in the other cases, let us consider the shortened expression

$$
\delta^{j_{n+1} i} \delta^{\left(k j_{1} \cdots j_{n} k_{1} \cdots k_{n} k_{n+1} \cdots\right)} \mu_{j_{1} \cdots j_{n+1}} \mu_{k_{1} \cdots k_{n+1}} .
$$

Here we can exchange the nomes of the indexes $j$. with those of the $k$., so that the above shortened expression becomes

$$
\delta^{k_{n+1} i} \delta^{\left(k k_{1} \cdots k_{n} j_{1} \cdots j_{n} j_{n+1} \cdots\right)} \mu_{k_{1} \cdots k_{n+1}} \mu_{j_{1} \cdots j_{n+1}} .
$$

Now we can exchange the indexes $k_{1} \cdots k_{n}$ with the indexes $j_{1} \cdots j_{n}$ in the expression of $\delta^{\left(k k_{1} \cdots k_{n} j_{1} \cdots j_{n} j_{n+1} \cdots\right)}$ because this is a symmetric tensor.

We obtain $\delta^{k_{n+1} i} \delta^{\left(k j_{1} \cdots j_{n} k_{1} \cdots k_{n} j_{n+1} \cdots\right)} \mu_{k_{1} \cdots k_{n+1}} \mu_{j_{1} \cdots j_{n+1}}$. By comparing this result with the expression which we started from, it is the same if he had exchanged the indexes $j_{n+1}$ and $k_{n+1}$. This completes the proof of the fact that the expression (7) remains the same if we exchange two indexes of the set $j_{1} \cdots j_{n+1} A_{n+1,1} \cdots A_{n+1, p_{n+1}-1}$; so we can insert there a symmetrization over those indexes and (7) becomes

$$
\begin{gathered}
\sum_{n=0}^{N+1}(n+1) \mu_{i j_{1} \cdots j_{n}} \frac{\partial h^{\prime}}{\partial \mu_{k j_{1} \cdots j_{n}}}=\sum_{n=0}^{N+1}(n+1) p_{n+1} \cdot \\
\sum_{p_{0}, \cdots, p_{N+2}, r_{1}, \cdots, r_{N}}^{0 \cdots \infty} \frac{1}{p_{0} !} \frac{1}{p_{1} !} \cdots \frac{1}{p_{N+2} !} \frac{1}{r_{1} !} \frac{1}{r_{2} !} \cdots \frac{1}{r_{N} !} \cdot \\
\sum_{i=1}^{N+2} i p_{i}+\sum_{i=1}^{N} i r_{i} e v e n \\
\frac{\left[1+\sum_{i=1}^{N+2} i p_{i}+\sum_{i=1}^{N} i r_{i}\right] ! !}{1+\sum_{i=1}^{N+2} i p_{i}+\sum_{i=1}^{N} i r_{i}} \frac{1+\sum_{i=0}^{N+2}(i-2) p_{i}+\sum_{i=1}^{N} i r_{i}}{\left[1+\sum_{i=0}^{N+2}(i-2) p_{i}+\sum_{i=1}^{N} i r_{i}\right] ! !} .
\end{gathered}
$$




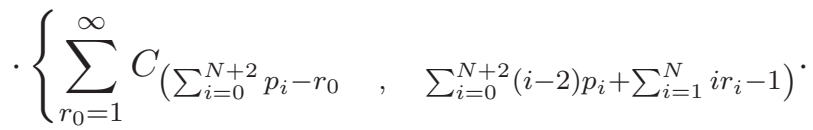

$$
\begin{aligned}
& \cdot \frac{\partial^{\sum_{i=1}^{N} r_{i}}}{\partial \lambda^{\sum_{i=1}^{N} r_{i}}}\left[\left(\frac{-1}{2 \lambda}\right)^{r_{0}}\right]+\sum_{r_{0}=0}^{\infty} \frac{1}{r_{0} !}(-2)^{\sum_{i=0}^{N} r_{i}}\left(\sum_{i=0}^{N} r_{i}\right) ! \cdot \\
& \left.\cdot C_{\left(\sum_{i=0}^{N+2} p_{i}+\sum_{i=0}^{N} r_{i} \quad, \quad \sum_{i=0}^{N+2}(i-2) p_{i}+\sum_{i=1}^{N} i r_{i}-1\right)}(\lambda)^{r_{0}}\right\} . \\
& \cdot \delta \underline{j_{n+1}} i \delta\left(\underline{j_{1} \cdots j_{n}} A_{1,1} \cdots A_{1, p_{1}} \cdots A_{n+1,1} \cdots A_{n+1, p_{n+1}-1} \cdots A_{N+2,1} \cdots A_{N+2, p_{N+2}}\right. \\
& \left.B_{1,1} \cdots B_{1, r_{1}} \cdots B_{N, 1} \cdots B_{N, r_{N}}\right) . \\
& \cdot(\mu)^{p_{0}} \mu_{A_{1,1}} \cdots \mu_{A_{1, p_{1}}} \cdots \mu_{A_{n+1,1}} \cdots \mu_{A_{n+1, p_{n+1}-1}} \mu_{j_{1} \cdots j_{n+1}} \cdots \\
& \mu_{A_{N+2,1}} \cdots \mu_{A_{N+2, p_{N+2}}} \lambda_{B_{1,1}} \cdots \lambda_{B_{1, r_{1}}} \cdots \lambda_{B_{N, 1}} \cdots \lambda_{B_{N, r_{N}}},
\end{aligned}
$$

where underlined indexes denote symmetrization over these indexes. Now we observe that $(n+1) p_{n+1}$ is exactly the number of the indexes of the set $j_{1} \cdots j_{n+1} A_{n+1,1} \cdots A_{n+1, p_{n+1}-1}$ and that, thanks to the summation $\sum_{n=0}^{N+1}$ the index near $i$ in $\delta^{j_{n+1} i}$ can be every index of the set

$A_{1,1} \cdots A_{1, p_{1}} \cdots A_{n+1,1} \cdots A_{n+1, p_{n+1}} \cdots A_{N+2,1} \cdots A_{N+2, p_{N+2}}$. These facts allow to rewrite (8) as

$$
\begin{gathered}
\sum_{n=0}^{N+1}(n+1) \mu_{i j_{1} \cdots j_{n}} \frac{\partial h^{\prime}}{\partial \mu_{k j_{1} \cdots j_{n}}}=\left[\sum_{n=0}^{N+1}(n+1) p_{n+1}\right] \\
\sum_{p_{0}, \cdots, p_{N+2}, r_{1}, \cdots, r_{N}}^{N \cdots \infty} \frac{1}{p_{0} !} \frac{1}{p_{1} !} \cdots \frac{1}{p_{N+2} !} \frac{1}{r_{1} !} \frac{1}{r_{2} !} \cdots \frac{1}{r_{N} !} . \\
\sum_{i=1}^{N+2} i p_{i}+\sum_{i=1}^{N} i r_{i} \text { even } \\
\frac{\left[1+\sum_{i=1}^{N+2} i p_{i}+\sum_{i=1}^{N} i r_{i}\right] ! !}{1+\sum_{i=1}^{N+2} i p_{i}+\sum_{i=1}^{N} i r_{i}} \frac{1+\sum_{i=0}^{N+2}(i-2) p_{i}+\sum_{i=1}^{N} i r_{i}}{\left[1+\sum_{i=0}^{N+2}(i-2) p_{i}+\sum_{i=1}^{N} i r_{i}\right] ! !} . \\
\cdot\left\{\sum_{r_{0}=1}^{\infty} C_{\left(\sum_{i=0}^{N+2} p_{i}-r_{0}\right.}, \quad \sum_{i=0}^{N+2}(i-2) p_{i}+\sum_{i=1}^{N} i r_{i}-1\right)
\end{gathered} .
$$




$$
\begin{aligned}
& \cdot \frac{\partial^{\sum_{i=1}^{N} r_{i}}}{\partial \lambda \sum_{i=1}^{N} r_{i}}\left[\left(\frac{-1}{2 \lambda}\right)^{r_{0}}\right]+\sum_{r_{0}=0}^{\infty} \frac{1}{r_{0} !}(-2)^{\sum_{i=0}^{N} r_{i}}\left(\sum_{i=0}^{N} r_{i}\right) ! \cdot \\
& \left.\cdot C_{\left(\sum_{i=0}^{N+2} p_{i}+\sum_{i=0}^{N} r_{i}\right.}, \quad \sum_{i=0}^{N+2}(i-2) p_{i}+\sum_{i=1}^{N} i r_{i}-1\right) \\
& \left.(\lambda)^{r_{0}}\right\} .
\end{aligned}
$$

$\cdot \delta \underline{j_{n+1} i} \delta \underline{\left(k j_{1} \cdots j_{n} A_{1,1} \cdots A_{1, p_{1}} \cdots A_{n+1,1} \cdots A_{n+1, p_{n+1}-1} \cdots A_{N+2,1} \cdots A_{N+2, p_{N+2}}\right.}$

$$
\left.B_{1,1} \cdots B_{1, r_{1}} \cdots B_{N, 1} \cdots B_{N, r_{N}}\right)
$$

$$
\begin{array}{r}
\cdot(\mu)^{p_{0}} \mu_{A_{1,1}} \cdots \mu_{A_{1, p_{1}}} \cdots \mu_{A_{n+1,1}} \cdots \mu_{A_{n+1, p_{n+1}-1}} \\
\cdot \mu_{j_{1} \cdots j_{n+1}} \cdots \mu_{A_{N+2,1}} \cdots \mu_{A_{N+2, p_{N+2}}} \lambda_{B_{1,1}} \cdots \lambda_{B_{1, r_{1}}} \cdots \lambda_{B_{N, 1}} \cdots \lambda_{B_{N, r_{N}}} .
\end{array}
$$

- For the fourth term of (6) we can do similar passages (the difference is that we have the $\lambda \ldots$ instead of the $\mu \ldots, N-2$ instead of $N$ and $s$ instead of $n$ ); in this way that term becomes

$$
\begin{gathered}
\sum_{s=0}^{N-1}(s+1) \lambda_{i h_{1} \cdots h_{s}} \frac{\partial h^{\prime}}{\partial \lambda_{k h_{1} \cdots h_{s}}}=\left[\sum_{s=0}^{N-1}(s+1) r_{s+1}\right] \\
\sum_{p_{0}, \cdots, p_{N+2}, r_{1}, \cdots, r_{N}}^{0 \cdots \infty} \frac{1}{p_{0} !} \frac{1}{p_{1} !} \cdots \frac{1}{p_{N+2} !} \frac{1}{r_{1} !} \frac{1}{r_{2} !} \cdots \frac{1}{r_{N} !} \\
\sum_{i=1}^{N+2} i p_{i}+\sum_{i=1}^{N} i r_{i} \text { even } \\
\frac{\left.1+\sum_{i=1}^{N+2} i p_{i}+\sum_{i=1}^{N} i r_{i}\right] ! !}{1+\sum_{i=1}^{N+2} i p_{i}+\sum_{i=1}^{N} i r_{i}} \frac{1+\sum_{i=0}^{N+2}(i-2) p_{i}+\sum_{i=1}^{N} i r_{i}}{\left[1+\sum_{i=0}^{N+2}(i-2) p_{i}+\sum_{i=1}^{N} i r_{i}\right] ! !} \\
\cdot\left\{\sum_{r_{0}=1}^{\infty} C_{\left(\sum_{i=0}^{N+2} p_{i}-r_{0}\right.}, \sum_{i=0}^{N+2}(i-2) p_{i}+\sum_{i=1}^{N} i r_{i}-1\right) \\
\cdot \frac{\partial \sum_{i=1}^{N} r_{i}}{\partial \lambda \sum_{i=1}^{N} r_{i}}\left[\left(\frac{-1}{2 \lambda}\right)^{r_{0}}\right]+\sum_{r_{0}=0}^{\infty} \frac{1}{r_{0} !}(-2)^{\sum_{i=0}^{N} r_{i}}\left(\sum_{i=0}^{N} r_{i}\right) ! \\
\left.\cdot C_{\left(\sum_{i=0}^{N+2} p_{i}+\sum_{i=0}^{N} r_{i}\right.}, \quad \sum_{i=0}^{N+2}(i-2) p_{i}+\sum_{i=1}^{N} i r_{i}-1\right) \\
\left.(\lambda)^{r_{0}}\right\}
\end{gathered}
$$




$$
\begin{array}{r}
\cdot \delta \frac{h_{s+1} i}{} \delta \underline{\left(k h_{1} \cdots h_{s}\right.} A_{1,1} \cdots A_{1, p_{1}} \cdots A_{N+2,1} \cdots A_{N+2, p_{N+2}} \\
\frac{\left.B_{1,1} \cdots B_{1, r_{1}} \cdots B_{s+1,1} \cdots B_{s+1, r_{s+1}-1} \cdots B_{N, 1} \cdots B_{N, r_{N}}\right)}{\cdot(\mu)^{p_{0}} \mu_{A_{1,1}} \cdots \mu_{A_{1, p_{1}}} \cdots \mu_{A_{N+2,1}} \cdots \mu_{A_{N+2, p_{N+2}}}} \\
\cdot \lambda_{B_{1,1}} \cdots \lambda_{B_{1, r_{1}}} \cdots \lambda_{B_{s+1,1}} \cdots \lambda_{B_{s+1, r_{s+1}-1}} \cdots \lambda_{B_{N, 1}} \cdots \lambda_{B_{N, r_{N}}}
\end{array} .
$$

- If we look at the last term of (6), we see that it can be written together with (9) and (10) and they become

$$
\begin{aligned}
& \sum_{n=0}^{N+1} \frac{\partial h^{\prime}}{\partial \mu_{k j_{1} \cdots j_{n}}}(n+1) \mu_{i j_{1} \cdots j_{n}}+\sum_{s=0}^{N-1} \frac{\partial h^{\prime}}{\partial \lambda_{k h_{1} \cdots h_{s}}}(s+1) \lambda_{i h_{1} \cdots h_{s}}+ \\
& +h^{\prime} \delta^{k i}=\left[1+\sum_{n=0}^{N+1}(n+1) p_{n+1}+\sum_{s=0}^{N-1}(s+1) r_{s+1}\right] \text {. } \\
& \sum^{0 \cdots \infty} \quad \frac{1}{p_{0} !} \frac{1}{p_{1} !} \cdots \frac{1}{p_{N+2} !} \frac{1}{r_{1} !} \frac{1}{r_{2} !} \cdots \frac{1}{r_{N} !} . \\
& p_{0}, \cdots, p_{N+2}, r_{1}, \cdots, r_{N} \\
& \sum_{i=1}^{N+2} i p_{i}+\sum_{i=1}^{N} i r_{i} \text { even } \\
& \frac{\left[1+\sum_{i=1}^{N+2} i p_{i}+\sum_{i=1}^{N} i r_{i}\right] ! !}{1+\sum_{i=1}^{N+2} i p_{i}+\sum_{i=1}^{N} i r_{i}} \frac{1+\sum_{i=0}^{N+2}(i-2) p_{i}+\sum_{i=1}^{N} i r_{i}}{\left[1+\sum_{i=0}^{N+2}(i-2) p_{i}+\sum_{i=1}^{N} i r_{i}\right] ! !} .
\end{aligned}
$$

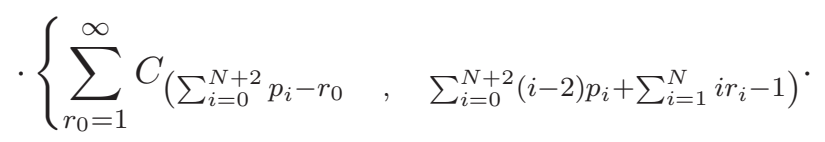

$$
\begin{aligned}
& \cdot \frac{\partial^{\sum_{i=1}^{N} r_{i}}}{\partial \lambda^{\sum_{i=1}^{N} r_{i}}}\left[\left(\frac{-1}{2 \lambda}\right)^{r_{0}}\right]+\sum_{r_{0}=0}^{\infty} \frac{1}{r_{0} !}(-2)^{\sum_{i=0}^{N} r_{i}}\left(\sum_{i=0}^{N} r_{i}\right) ! \cdot \\
& \left.\cdot C_{\left(\sum_{i=0}^{N+2} p_{i}+\sum_{i=0}^{N} r_{i} \quad, \quad \sum_{i=0}^{N+2}(i-2) p_{i}+\sum_{i=1}^{N} i r_{i}-1\right)}(\lambda)^{r_{0}}\right\} . \\
& \left.\cdot \delta \underline{\underline{k}} i \delta \underline{\left(A_{1,1} \cdots A_{1, p_{1}} \cdots A_{N+2,1} \cdots A_{N+2, p_{N+2}} B_{1,1} \cdots B_{1, r_{1}} \cdots B_{N, 1} \cdots B_{N, r_{N}}\right.}\right) .
\end{aligned}
$$




$$
\begin{array}{r}
\cdot(\mu)^{p_{0}} \mu_{A_{1,1}} \cdots \mu_{A_{1, p_{1}}} \cdots \mu_{A_{N+2,1}} \cdots \mu_{A_{N+2, p_{N+2}}} . \\
\cdot \lambda_{B_{1,1}} \cdots \lambda_{B_{1, r_{1}}} \cdots \lambda_{B_{N, 1}} \cdots \lambda_{B_{N, r_{N}}},
\end{array}
$$

where also the index $k$ has been put under the symmetrization and we recall that a property of symmetrization is $\delta^{i \underline{k}} \delta^{(\cdots)}=\delta^{i \underline{k}} \delta \cdots=\delta^{(i k \cdots)}$. It is interesting to see that the coefficient in square bracket at the beginning of the right hand side of eq. (11) has become equal to the denominator of the half factorial of that right hand side!

- For the second term of (6), we can use (4) and the derivation causes someway the rising of one unity of the index $p_{2}$; more precisely we have

$$
\begin{aligned}
& \frac{\partial h^{\prime}}{\partial \mu_{k i}} 2 \lambda= \\
& =2 \lambda \quad \sum^{0 \cdots \infty} \quad \frac{1}{p_{0} !} \frac{1}{p_{1} !} \cdots \frac{1}{p_{N+2} !} \frac{1}{r_{1} !} \frac{1}{r_{2} !} \cdots \frac{1}{r_{N} !} . \\
& p_{0}, \cdots, p_{N+2}, r_{1}, \cdots, r_{N} \\
& \sum_{i=1}^{N+2} i p_{i}+\sum_{i=1}^{N} i r_{i} \text { even } \\
& \cdot\left[1+\sum_{i=1}^{N+2} i p_{i}+\sum_{i=1}^{N} i r_{i}\right] ! ! \frac{1+\sum_{i=0}^{N+2}(i-2) p_{i}+\sum_{i=1}^{N} i r_{i}}{\left[1+\sum_{i=0}^{N+2}(i-2) p_{i}+\sum_{i=1}^{N} i r_{i}\right] ! !} .
\end{aligned}
$$

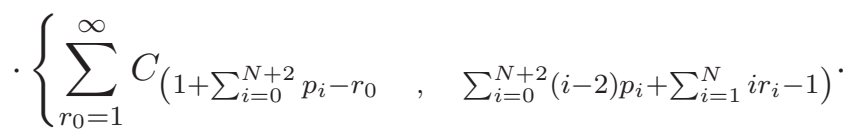

$$
\begin{aligned}
& \cdot \frac{\partial^{\sum_{i=1}^{N} r_{i}}}{\partial \lambda^{\sum_{i=1}^{N} r_{i}}}\left[\left(\frac{-1}{2 \lambda}\right)^{r_{0}}\right]+\sum_{r_{0}=0}^{\infty} \frac{1}{r_{0} !}(-2)^{\sum_{i=0}^{N} r_{i}}\left(\sum_{i=0}^{N} r_{i}\right) ! \cdot \\
& \text { - } \left.C_{\left(1+\sum_{i=0}^{N+2} p_{i}+\sum_{i=0}^{N} r_{i} \quad, \quad \sum_{i=0}^{N+2}(i-2) p_{i}+\sum_{i=1}^{N} i r_{i}-1\right)}(\lambda)^{r_{0}}\right\} \text {. } \\
& \cdot \delta^{\left(k i A_{1,1} \cdots A_{1, p_{1}} \cdots A_{N+2,1} \cdots A_{N+2, p_{N+2}} B_{1,1} \cdots B_{1, r_{1}} \cdots B_{N, 1} \cdots B_{N, r_{N}}\right)} . \\
& \cdot(\mu)^{p_{0}} \mu_{A_{1,1}} \cdots \mu_{A_{1, p_{1}}} \cdots \mu_{A_{N+2,1}} \cdots \mu_{A_{N+2, p_{N+2}}} \cdot \\
& \cdot \lambda_{B_{1,1}} \cdots \lambda_{B_{1, r_{1}}} \cdots \lambda_{B_{N, 1}} \cdots \lambda_{B_{N, r_{N}}},
\end{aligned}
$$


- There remains to consider the third term of (6); for it we can use (4) and the derivation causes someway the rising of one unity of the index $p_{s+3}$; more precisely we have

$$
\begin{aligned}
& \sum_{s=0}^{N-1} 2 \lambda_{j_{1} \cdots j_{s+1}} \frac{\partial h^{\prime}}{\partial \mu_{k j_{1} \cdots j_{s+1} i}}=\sum_{s=0}^{N-1} 2 \lambda_{j_{1} \cdots j_{s+1}} \\
& \sum^{0 \cdots \infty} \quad \frac{1}{p_{0} !} \frac{1}{p_{1} !} \cdots \frac{1}{p_{N+2} !} \frac{1}{r_{1} !} \frac{1}{r_{2} !} \cdots \frac{1}{r_{N} !} . \\
& p_{0}, \cdots, p_{N+2}, r_{1}, \cdots, r_{N} \\
& s+3+\sum_{i=1}^{N+2} i p_{i}+\sum_{i=1}^{N} i r_{i} \text { even } \\
& \cdot \frac{\left[s+4+\sum_{i=1}^{N+2} i p_{i}+\sum_{i=1}^{N} i r_{i}\right] ! !}{s+4+\sum_{i=1}^{N+2} i p_{i}+\sum_{i=1}^{N} i r_{i}} . \\
& \cdot \frac{1}{\left[s+\sum_{i=0}^{N+2}(i-2) p_{i}+\sum_{i=1}^{N} i r_{i}\right] ! !} \text {. } \\
& \cdot\left\{\sum_{r_{0}=1}^{\infty} C_{\left(1+\sum_{i=0}^{N+2} p_{i}-r_{0}\right.} \quad, \quad \sum_{i=0}^{N+2}(i-2) p_{i}+\sum_{i=1}^{N} i r_{i}+s\right)^{+} \\
& \cdot \frac{\partial^{\sum_{i=1}^{N} r_{i}}}{\partial \lambda^{\sum_{i=1}^{N} r_{i}}}\left[\left(\frac{-1}{2 \lambda}\right)^{r_{0}}\right]+\sum_{r_{0}=0}^{\infty} \frac{1}{r_{0} !}(-2)^{\sum_{i=0}^{N} r_{i}}\left(\sum_{i=0}^{N} r_{i}\right) ! \cdot \\
& \left.\cdot C_{\left(1+\sum_{i=0}^{N+2} p_{i}+\sum_{i=0}^{N} r_{i} \quad, \quad \sum_{i=0}^{N+2}(i-2) p_{i}+\sum_{i=1}^{N} i r_{i}+s\right)}(\lambda)^{r_{0}}\right\} . \\
& \cdot \delta^{\left(k j_{1} \cdots j_{s+1} i A_{1,1} \cdots A_{1, p_{1}} \cdots A_{N+2,1} \cdots A_{N+2, p_{N+2}} B_{1,1} \cdots B_{1, r_{1}} \cdots B_{N, 1} \cdots B_{N, r_{N}}\right)}(\mu)^{p_{0}} \cdot \\
& \cdot \mu_{A_{1,1}} \cdots \mu_{A_{1, p_{1}}} \cdots \mu_{A_{N+2,1}} \cdots \mu_{A_{N+2, p_{N+2}}} \cdot \\
& \cdot \lambda_{B_{1,1}} \cdots \lambda_{B_{1, r_{1}}} \cdots \lambda_{B_{N, 1}} \cdots \lambda_{B_{N, r_{N}}}
\end{aligned}
$$

In this expression, we can substitute $\lambda_{j_{1} \cdots j_{s+1}}$ with $\lambda_{B_{s+1, r_{s+1}+1}}$ and $j_{1} \cdots j_{s+1}$ with $B_{s+1, r_{s+1}+1}$; moreover, we substitute $\frac{1}{r_{s+1} !}$ with $\frac{r_{s+1}+1}{\left(r_{s+1}+1\right) !}$ and, after that, decrease $r_{s+1}$ of one unity (which is equivalent to a 
change of index). So this expression becomes

$$
\begin{aligned}
& \sum_{s=0}^{N-1} 2 \lambda_{j_{1} \cdots j_{s+1}} \frac{\partial h^{\prime}}{\partial \mu_{k j_{1} \cdots j_{s+1} i}}=\sum_{s=0}^{N-1} 2 r_{s+1} \\
& \sum^{0 \cdots \infty} \quad \frac{1}{p_{0} !} \frac{1}{p_{1} !} \cdots \frac{1}{p_{N+2} !} \frac{1}{r_{1} !} \frac{1}{r_{2} !} \cdots \frac{1}{r_{N} !} . \\
& p_{0}, \cdots, p_{N+2}, r_{1}, \cdots, r_{N} \\
& 2+\sum_{i=1}^{N+2} i p_{i}+\sum_{i=1}^{N} i r_{i} \text { even } \\
& \cdot \frac{\left[1+\sum_{i=1}^{N+2} i p_{i}+\sum_{i=1}^{N} i r_{i}\right] ! !}{\left[-1+\sum_{i=0}^{N+2}(i-2) p_{i}+\sum_{i=1}^{N} i r_{i}\right] ! !}
\end{aligned}
$$

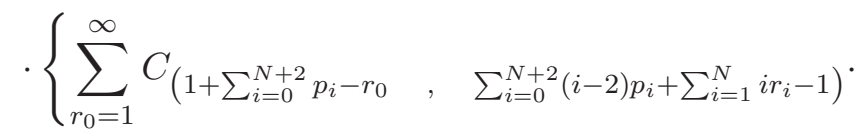

$$
\begin{aligned}
& \cdot \frac{\partial^{-1+\sum_{i=1}^{N} r_{i}}}{\partial \lambda^{-1+\sum_{i=1}^{N} r_{i}}}\left[\left(\frac{-1}{2 \lambda}\right)^{r_{0}}\right]+\sum_{r_{0}=0}^{\infty} \frac{1}{r_{0} !}(-2)^{-1+\sum_{i=0}^{N} r_{i}}\left(-1+\sum_{i=0}^{N} r_{i}\right) ! \cdot \\
& \left.\cdot C_{\left(\sum_{i=0}^{N+2} p_{i}+\sum_{i=0}^{N} r_{i} \quad, \quad \sum_{i=0}^{N+2}(i-2) p_{i}+\sum_{i=1}^{N} i r_{i}-1\right)}(\lambda)^{r_{0}}\right\} \text {. } \\
& \cdot \delta^{\left(k i A_{1,1} \cdots A_{1, p_{1}} \cdots A_{N+2,1} \cdots A_{N+2, p_{N+2}} B_{1,1} \cdots B_{1, r_{1}} \cdots B_{N, 1} \cdots B_{N, r_{N}}\right)}(\mu)^{p_{0}} . \\
& \cdot \mu_{A_{1,1}} \cdots \mu_{A_{1, p_{1}}} \cdots \mu_{A_{N+2,1}} \cdots \mu_{A_{N+2, p_{N+2}}} . \\
& \lambda_{B_{1,1}} \cdots \lambda_{B_{1, r_{1}}} \cdots \lambda_{B_{N, 1}} \cdots \lambda_{B_{N, r_{N}}} .
\end{aligned}
$$

It is true that with change of index we have in the summation the extra term with $r_{s+1}=0$; but it doesn't effect the result for the presence of the coefficient $r_{s+1}$.

If we look at the expressions (11), (12) and (14) we conclude that to prove (6) it is sufficient that the following relation holds

$$
\left.0=\sum_{r_{0}=1}^{\infty} C_{\left(\sum_{i=0}^{N+2} p_{i}-r_{0}\right.} \quad, \quad \sum_{i=0}^{N+2}(i-2) p_{i}+\sum_{i=1}^{N} i r_{i}-1\right)
$$




$$
\begin{aligned}
& \cdot \frac{\partial^{\sum_{i=1}^{N} r_{i}}}{\partial \lambda^{\sum_{i=1}^{N} r_{i}}}\left[\left(\frac{-1}{2 \lambda}\right)^{r_{0}}\right]+\sum_{r_{0}=0}^{\infty} \frac{1}{r_{0} !}(-2)^{\sum_{i=0}^{N} r_{i}}\left(\sum_{i=0}^{N} r_{i}\right) ! \cdot \\
& \cdot C_{\left(\sum_{i=0}^{N+2} p_{i}+\sum_{i=0}^{N} r_{i} \quad, \quad \sum_{i=0}^{N+2}(i-2) p_{i}+\sum_{i=1}^{N} i r_{i}-1\right)}(\lambda)^{r_{0}}+ \\
& \left.+2 \lambda \sum_{r_{0}=1}^{\infty} C_{\left(1+\sum_{i=0}^{N+2} p_{i}-r_{0}\right.} \quad, \quad \sum_{i=0}^{N+2}(i-2) p_{i}+\sum_{i=1}^{N} i r_{i}-1\right) . \\
& \cdot \frac{\partial^{\sum_{i=1}^{N} r_{i}}}{\partial \lambda^{\sum_{i=1}^{N} r_{i}}}\left[\left(\frac{-1}{2 \lambda}\right)^{r_{0}}\right]+2 \lambda \sum_{r_{0}=0}^{\infty} \frac{1}{r_{0} !}(-2)^{\sum_{i=0}^{N} r_{i}}\left(\sum_{i=0}^{N} r_{i}\right) ! \\
& \cdot C_{\left(1+\sum_{i=0}^{N+2} p_{i}+\sum_{i=0}^{N} r_{i} \quad, \quad \sum_{i=0}^{N+2}(i-2) p_{i}+\sum_{i=1}^{N} i r_{i}-1\right)}(\lambda)^{r_{0}}+ \\
& +\sum_{s=0}^{N-1} 2 r_{s+1}\left\{\sum_{r_{0}=1}^{\infty} C_{\left(1+\sum_{i=0}^{N+2} p_{i}-r_{0}\right.} \quad, \quad-1+\sum_{i=0}^{N+2}(i-2) p_{i}+\sum_{i=1}^{N} i r_{i}\right) . \\
& \cdot \frac{\partial^{-1+\sum_{i=1}^{N} r_{i}}}{\partial \lambda^{-1+\sum_{i=1}^{N} r_{i}}}\left[\left(\frac{-1}{2 \lambda}\right)^{r_{0}}\right]+\sum_{r_{0}=0}^{\infty} \frac{1}{r_{0} !}(-2)^{-1+\sum_{i=0}^{N} r_{i}}\left(-1+\sum_{i=0}^{N} r_{i}\right) ! \cdot \\
& \left.\cdot C_{\left(\sum_{i=0}^{N+2} p_{i}+\sum_{i=0}^{N} r_{i} \quad, \quad \sum_{i=0}^{N+2}(i-2) p_{i}+\sum_{i=1}^{N} i r_{i}-1\right)}(\lambda)^{r_{0}}\right\} .
\end{aligned}
$$

Now the terms in lines 3,5 of this relation can be written together as

$$
\begin{aligned}
& \frac{\partial^{\sum_{i=1}^{N} r_{i}}}{\partial \lambda^{\sum_{i=1}^{N} r_{i}}}\left[2 \lambda \sum_{r_{0}=1}^{\infty} C_{\left(1+\sum_{i=0}^{N+2} p_{i}-r_{0}\right.} \quad, \quad-1+\sum_{i=0}^{N+2}(i-2) p_{i}+\sum_{i=1}^{N} i r_{i}\right) . \\
& \left.\cdot\left(\frac{-1}{2 \lambda}\right)^{r_{0}}\right]= \\
& =-\frac{\partial^{\sum_{i=1}^{N} r_{i}}}{\partial \lambda^{\sum_{i=1}^{N} r_{i}}}\left[\sum_{r_{0}=1}^{\infty} C_{\left(1+\sum_{i=0}^{N+2} p_{i}-r_{0}\right.} \quad, \quad-1+\sum_{i=0}^{N+2}(i-2) p_{i}+\sum_{i=1}^{N} i r_{i}\right) . \\
& \left.\cdot\left(\frac{-1}{2 \lambda}\right)^{r_{0}-1}\right]= \\
& =-\frac{\partial^{\sum_{i=1}^{N} r_{i}}}{\partial \lambda^{\sum_{i=1}^{N} r_{i}}}\left[\sum_{\psi=0}^{\infty} C_{\left(\sum_{i=0}^{N+2} p_{i}-\psi\right.}, \quad-1+\sum_{i=0}^{N+2}(i-2) p_{i}+\sum_{i=1}^{N} i r_{i}\right) .
\end{aligned}
$$




$$
\begin{array}{r}
\left.\cdot\left(\frac{-1}{2 \lambda}\right)^{\psi}\right]= \\
=-\frac{\partial^{\sum_{i=1}^{N} r_{i}}}{\partial \lambda^{N} \sum_{i=1}^{N} r_{i}}\left[\sum_{\psi=1}^{\infty} C_{\left(\sum_{i=0}^{N+2} p_{i}-\psi,-1+\sum_{i=0}^{N+2}(i-2) p_{i}+\sum_{i=1}^{N} i r_{i}\right)}\left(\frac{-1}{2 \lambda}\right)^{\psi}\right]
\end{array}
$$

where in the second passage we have changed index with the law $r_{0}=\psi+1$ and in the last passage we have excluded the value with $\psi=0$ because the derivative of a constant is zero; obviously this excludes the case with $r_{1}+r_{2}+\cdots+r_{N}=0$. The result is exactly the opposite of the first term in eq. (15) in the case $r_{1}+r_{2}+\cdots+r_{N} \neq 0$. Instead of this, if $r_{1}+r_{2}+\cdots+r_{N}=0$ (that is $r_{1}=0$, $r_{2}=0, \cdots, r_{N}=0$ ) the term in line 5 of eq. (15) is not present and, while those in lines 1,3 reduce to

$$
\begin{array}{r}
\left.0=\sum_{r_{0}=1}^{\infty} C_{\left(\sum_{i=0}^{N+2} p_{i}-r_{0}\right.}, \sum_{i=0}^{N+2}(i-2) p_{i}-1\right)\left(\frac{-1}{2 \lambda}\right)^{r_{0}}+ \\
\left.-\sum_{r_{0}=1}^{\infty} C_{\left(1+\sum_{i=0}^{N+2} p_{i}-r_{0}\right.} \quad, \quad \sum_{i=0}^{N+2}(i-2) p_{i}-1\right) \\
\left.=-C_{\left(\sum_{i=0}^{N+2} p_{i}\right.}, \quad \sum_{i=0}^{N+2}\right)^{r_{0}-1}= \\
\end{array}
$$

where in the second passage we have changed the index of the second summation with the law $r_{0}=\psi+1$.

So, up to now, we have found that the terms in lines 1, 3, 5 of eq. (15) are present only if $r_{1}=0, r_{2}=0, \cdots, r_{N}=0$ and, in this case, their sum is

$$
-C_{\left(\sum_{i=0}^{N+2} p_{i} \quad, \quad \sum_{i=0}^{N+2}(i-2) p_{i}-1\right)} .
$$

Now the term in line 4 of eq. (15) can be transformed by substituting $\frac{1}{r_{0} !}$ with $\frac{r_{0}+1}{\left(r_{0}+1\right) !}$ and then decreasing $r_{0}$ of an unity; in this way it becomes

$$
\begin{array}{r}
2 \sum_{r_{0}=0}^{\infty} \frac{r_{0}}{r_{0} !}(-2)^{-1+\sum_{i=0}^{N} r_{i}}\left(-1+\sum_{i=0}^{N} r_{i}\right) ! \\
\cdot C_{\left(\sum_{i=0}^{N+2} p_{i}+\sum_{i=0}^{N} r_{i} \quad, \quad \sum_{i=0}^{N+2}(i-2) p_{i}+\sum_{i=1}^{N} i r_{i}-1\right)}(\lambda)^{r_{0}} .
\end{array}
$$

It is true that int this way we have added the term with $r_{0}=0$, but this term gives no contribute, thanks to the factor $r_{0}$. The result, jointly with the term 
in line 6 of eq. (15) becomes

$$
\begin{array}{r}
2 \sum_{r_{0}=0}^{\infty} \frac{1}{r_{0} !}(-2)^{-1+\sum_{i=0}^{N} r_{i}}\left(\sum_{s=0}^{N} r_{s}\right) \cdot\left(-1+\sum_{i=0}^{N} r_{i}\right) ! \cdot \\
\cdot C_{\left(\sum_{i=0}^{N+2} p_{i}+\sum_{i=0}^{N} r_{i}, \sum_{i=0}^{N+2}(i-2) p_{i}+\sum_{i=1}^{N} i r_{i}-1\right)}(\lambda)^{r_{0}} .
\end{array}
$$

Consequently, the sum of the elements in lines $2,4,6$ of eq. (15) is

$$
\begin{array}{r}
\sum_{r_{0}=0}^{\infty} \frac{1}{r_{0} !}(-2)^{\sum_{i=0}^{N} r_{i}}\left[\left(\sum_{i=0}^{N} r_{i}\right) !-\left(\sum_{s=0}^{N} r_{s}\right) \cdot\left(-1+\sum_{i=0}^{N} r_{i}\right) !\right] \\
\left.\cdot C_{\left(\sum_{i=0}^{N+2} p_{i}+\sum_{i=0}^{N} r_{i}\right.}, \quad \sum_{i=0}^{N+2}(i-2) p_{i}+\sum_{i=1}^{N} i r_{i}-1\right)
\end{array}
$$

which is zero if $r_{1}+r_{2}+\cdots+r_{N} \neq 0$, while it is equal to

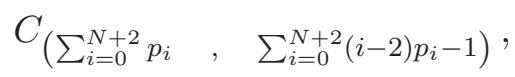

if $r_{1}=0, r_{2}=0, \cdots, r_{N}=0$, as a consequence of the identity

$$
r !-r(r-1) !=\left\{\begin{array}{ll}
0 & r>0 \\
1 & r=0
\end{array} .\right.
$$

So we have found that the sum of the lines 2, 4, 6 of eq. (15) is the opposite of the sum of its lines 1, 3, 5. This concludes the proof of eq. (15) and of all the arguments which we had to prove.

\section{Comparison with Previously Known Results}

- Let us begin with $N=0$, that is the eleven moments model.

We see that in the expression (4) of $h^{\prime}$, the second index of $C_{\text {., }}$ is negative so that we have $h^{\prime}=0$.

In the expression (5) of $h^{\prime k}$, the second index of $C_{.,}$. is not negative iff $p_{0}=0$, $p_{1}=0$ so that $\sum_{i=1}^{2} i p_{i}$ cannot be odd; it follows $h^{\prime k}=0$.

These results confirm the closure found in [17] where the contribute (4) and (5) for $h^{\prime}$ and $h^{\prime k}$ is not present.

- Let us consider the case $N=1$, that is the 24 moments model. 
We firstly note that in (4) and (5) we have to impose that the second index of $C$., must be non negative. For this reason $\sum_{p_{0}=0}^{\infty}$ transforms in $\sum_{p_{0}=0}^{\frac{r+s-p-2}{2}}$ in the expression for $h^{\prime}$ which is present in eq. (27) of [17] and in $\sum_{p_{0}=0}^{\frac{r+s-p-1}{2}}$ in the expression for $h^{\prime k}$ which is present in eq. (26) of [17].

After that, we note that in the line 4 of the present eq. (4) is present the term

$C_{p_{0}+p_{1}+p_{2}+p_{3}+r_{0}+r_{1},-2 p_{0}-p_{1}+p_{3}+r_{1}-1}$ whose first index is greater than the second one. Then this term is zero. There remains only the term in line 3 . In order not to have the first index negative we have to impose $r_{0} \leq p_{0}+p_{1}+p_{2}+p_{3}$; in order not to have the first index greater than the second one, we have to impose $r_{0} \geq 3 p_{0}+2 p_{1}+p_{2}-r_{1}+1$. For this reason $\sum_{r_{0}=1}^{\infty}$ transforms in $\sum_{\vartheta=\max \left\{1,3 p_{0}+2 p_{1}+p_{2}-r_{1}+1\right.}^{p_{0}+p_{1}+p_{2}+p_{3}}$ in the expression for $h^{\prime}$ which is present in eq. (27) of $[17]$.

For what concerns $h^{\prime k}$, we note that in the line 4 of the present eq. (5) is present a term $C$., whose first index is not greater than the second one only if $p_{0}=0, p_{1}=0, p_{2}=0, r_{0}=0$ so that $\sum_{i=1}^{N+2} i p_{i}+\sum_{i=1}^{N} i r_{i}=3 p_{3}+r_{1}$. It is easy to identify this term with that in the last line, eq. (26) of [17] with

$$
F_{r, s}=\frac{(r+3 s) ! !}{(r+s) ! !}(-2)^{r} r ! C_{r+s, r+s} .
$$

For what concerns the term in line 3 , in order not to have the first index negative we have to impose $r_{0} \leq p_{0}+p_{1}+p_{2}+p_{3}$; in order not to have the first index greater than the second one, we have to impose $r_{0} \geq 3 p_{0}+2 p_{1}+p_{2}-r_{1}$. For this reason $\sum_{r_{0}=1}^{\infty}$ transforms in $\sum_{\vartheta=\max \left\{1,3 p_{0}+2 p_{1}+p_{2}-r_{1}\right.}^{p_{0}+p_{1}+p_{2}+p_{3}}$ in the expression for $h^{\prime k}$ which is present in eq. (26) of [17].

Under every other aspect, the present (4) and (5) are the same thing than (27) and (26) of [17].

So in the present article we have seen how these terms originated from the 1-Model are transmitted also to the models with subsequent values of $N$.

Conclusions: In this paper we have found the general exact solution for the macroscopic approach to extended thermodynamics of polyatomic gases with many moments. In fact, we can take for $h^{\prime}$ the sum of the present expression (4), of the expression (4) of [2] and of (5) in [4]. Similarly, we can take for $h^{\prime k}$ the sum of the present expression (5), of the expression (5) of [2] and of (6) in [4]. The result is also a solution of the conditions (3) and, in the particular case $N=1$, it is equal to (4) and (5) of [2]. There is no possible further additional term for $h^{\prime}$ and $h^{\prime k}$, as outlined in the conclusions of [1]. This confirms the fact 
that the present results completely determine the requested solution for $h^{\prime}$ and $h^{\prime k}$.

\section{References}

[1] M.C. Carrisi, S. Pennisi, J.M. Sellier, Extended Thermodynamics of Dense Gases with many moments - The macroscopic approach, Int. Journal of Non-Linear Mech., 84 (2016), 12-22, doi: 10.1016/ijnonlinmec.2016.04.003.

[2] M.C. Carrisi, R. E. Tchame, M. Obounou, S. Pennisi, An exact solution for the macroscopic approach to extended Thermodynamic of Dense gases with many moments, $I J$ PAM., 106 (2016), 171-189, doi: 10.12732/ijpam.v106i1.13.

[3] M.C. Carrisi, S. Pennisi, J.M. Sellier, A kinetic type exact solution for Extended Thermodynamics of Dense Gases with many Moments, Journal of Computational and Theoretical Transport., 45 (2016), 162-173, doi: 10.1080/23324309.2016.1149079.

[4] M.C. Carrisi, R. Enoh Tchame, M. Obounou, S. Pennisi, A numberable Set of Exact Solutions for the Macroscopic Approach to Extended Thermodynamics of Polyatomic Gases with Many Moments, Advances in Mathematical Physics., 17 (2016), doi: 10.1155/2016/1307813.

[5] T. Arima, A. Mentrelli, T. Ruggeri, Molecular Extended Thermodynamics of rarefied polyatomic gases and wave velocities for increasing number of moments, Ann. Phys., 345 (2014), 111-132, doi: 10.1016/j.aop.2014.03.011.

[6] G. Boillat, T. Ruggeri, Hyperbolic principal subsystems: Entropy convexity and subcharacteristic conditions, Arch. Rat. Mech. Anal., 137 (1997), 305-320, doi: $10.1007 / \mathrm{s} 002050050030$.

[7] I-S. Liu, I. Müller, Extended Thermodynamics of Classical and Degenerate Ideal Gases, Arch. Rat. Mech. Anal., 83 (1993), 285-332, doi: 10.1007/BF00963838.

[8] I-S. Liu, I. Müller, T. Ruggeri, Relativistic thermodynamics of gases, Ann. Phys. (N.Y.), 169 (1986), 191-219, doi: 10.1016/0003-4916(86)90164-8.

[9] I. Müller, T. Ruggeri, Rational Extended Thermodynamics, 2nd edn. Springer Tracts in Natural Philosophy. Springer, New York (1998), doi: 10.1007/978-1-4612-2210-1.

[10] T. Arima, S. Taniguchi, T. Ruggeri, M. Sugiyama, Extended Thermodynamics of dense gases, Continuum Mech. Thermodyn., 24 (2012), 271-292, doi: 10.1007/s00161-011-0213$\mathrm{x}$.

[11] T. Ruggeri, M. Sugiyama, Rational Extended Thermodynamics beyond the Monatomic Gas, Springer Verlag (2015), doi: 10.1007/978-3-319-13341-6.

[12] T. Arima, T. Ruggeri, M. Sugiyama, S. Taniguchi, Monatomic gas as a singular limit of polyatomic gas in molecular extended thermodynamics with many moments, Annals of Physics, 372 (2016), 83-109, doi: 10.1016/j.aop.2016.04.015.

[13] M.C. Carrisi, S. Pennisi, T. Ruggeri, M. Sugiyama, Extended thermodynamics of dense gases in the presence of dynamic pressure, Ricerche di Matematica, 64 (2015), 403-419, doi: $10.1007 / \mathrm{s} 11587-015-0247-7$.

[14] M.C. Carrisi, S. Pennisi, An 18 Moments Model for Dense Gases: Entropy and Galilean Relativity Principles without Expansions, Entropy., 17 (2015), 214-230, doi: $10.3390 / \mathrm{e} 17010214$. 
[15] M.C. Carrisi, S. Pennisi, Extended thermodynamics for dense gases up to whatever order, Int. Journal of Non-Linear Mech., 77 (2015), 74-84, doi: 10.1016/ijnonlinmec.2015.07.011.

[16] M.C. Carrisi, R. E. Tchame, M. Obounou, S. Pennisi, Extended Thermodynamics for dense gases up to whatever order and with only some symmetries, Entropy., 17 (2015), 7052-7075, doi: 10.3390/e17107052.

[17] M.C. Carrisi, S. Pennisi, J.M. Sellier, Extended Thermodynamics of Dense Gases with at least 24 Moments, Ricerche di Matematica, 65 (2016), 505-522, doi: 10.1007/s11587016-02171-2.

[18] I-S. Liu, Method of Lagrange Multipliers for Exploitation of the Entropy Principle, Arch. Rat. Mech. Anal., 46 (1972), 131-148, doi: 10.1007/BF00250688.

[19] T. Ruggeri, A. Strumia, Main Field and Convex Covariant Density for Quasi-Linear Hyperbolic Systems. Relativistic Fluid Dynamics, Ann. Inst. H. Poincaré., 34 (1981), 65-84. 
